\title{
Surface Enhanced Raman Scattering with gold nanoparticles: Effect of particle shape
}

\author{
Furong Tian \\ Technological University Dublin, furong.tian@tudublin.ie \\ Franck Bonnier \\ Technological University Dublin, Franck.Bonnier@tudublin.ie \\ Alan Casey \\ Technological University Dublin, alan.casey@tudublin.ie
}

See next page for additional authors

Follow this and additional works at: https://arrow.tudublin.ie/nanolart

\section{Recommended Citation}

Tian, F. et al. (2014)Surface Enhanced Raman Scattering with gold nanoparticles: Effect of particle shape, Analytical Methods, 6, pp.9116-9123. doi:10.1039/C4AY02112F

This Article is brought to you for free and open access by the NanoLab at ARROW@TU Dublin. It has been accepted for inclusion in Articles by an authorized administrator of ARROW@TU Dublin. For more information, please contact arrow.admin@tudublin.ie, aisling.coyne@tudublin.ie,gerard.connolly@tudublin.ie. Funder: EU Marie Curie IF 


\section{Authors}

Furong Tian, Franck Bonnier, Alan Casey, Anne Shanahan, and Hugh Byrne

This article is available at ARROW@TU Dublin: https://arrow.tudublin.ie/nanolart/55 


\title{
Paper
}

Cite this: DOI: $10.1039 / x 0 x x 00000 x$

\section{Surface Enhanced Raman Scattering with gold nanoparticles: Effect of particle shape}

\author{
Furong Tian*, Franck Bonnier, Alan Casey, Anne E. Shanahan, Hugh J. Byrne
}

Received 00th January $x x x x$, Accepted 00th January $x x x x$

DOI: $10.1039 / \times 0 \times x 00000 x$

The dependence of the Surface Enhanced Raman Scattering (SERS) by gold nanoparticles on their shape is examined using the organic dye, rhodamine 6G (R6G) as probe molecule. SERS has been explored extensively for applications in sensing and imaging, but the design and optimisation of efficient substrates is still challenging. In order to understand and optimise the SERS process in nanoparticles, gold nanospheres and their aggregates, nanotriangles, and nanostars of similar dimensions were synthesised and characterised according to their average size, zeta potential and UV/visible absorption. SERS from R6G was negligible for unaggregated nanospheres at $532 \mathrm{~nm}$, close to the maximum of the surface plasmon resonance (SPR) at $560 \mathrm{~nm}$. Upon aggregation of the nanospheres, the SPR shifts to $\sim 660 \mathrm{~nm}$, attributable to local surface plasmon "hotspots" between the spheres, and the SERS signal of R6G is significantly increased, at $785 \mathrm{~nm}$. In monodisperse gold nanotriangles, the SPR is located at $\sim 800 \mathrm{~nm}$, and significant SERS of R6G is observed using $785 \mathrm{~nm}$ as source, as is the case for gold nanostars, which exhibit a double SPR with maxima at $\sim 600 \mathrm{~nm}$ and $\sim 785 \mathrm{~nm}$, attributable to the core sphere and vertices of the structures, respectively. In suspensions of equal nanoparticle and dye concentration, the SERS effect increases as nanospheres<nanosphere aggregates $<$ nanotriangles $<$ nanostars, clearly indicating that control over the number of local field hotspots can optimise the SERS efficiency. Notably, it is demonstrated that the SERS intensity per nanoparticle scales with the magnitude of the SPR absorbance at the excitation wavelength $(785 \mathrm{~nm})$, providing a clear guide to optimisation of the process experimentally.

\section{Introduction}

Gold nanoparticles (GNPs) are biocompatible and have advantageous optical properties for biomedical applications ${ }^{1}$. GNPs with different geometry, such as spheres, rods, triangles, hexagons, prisms, urchins, cubes, wires and stars have been explored for specific biomedical applications in dispersed form ${ }^{2}$. In this context, surface-enhanced Raman scattering/spectroscopy (SERS) has attracted a great deal of attention as a sensitive technique for chemical and bioanalytical sensing and imaging 2,3 . There exists a plethera of work which has been performed to demonstrate SERS effects for different molecules, with different shaped nanoparticles, at different dosages ${ }^{4}$, so much so that it is not easy to establish the principle parameters which need to be optimised for effective and reproducible SERS. The development of reliable quantitative comparisons is a priority which is required for meaningful design strategies for new nanomaterials. The purpose of this study is to identify critical conditions and physical properties of the materials which play the main role in optimised SERS.

Address, FOCAS Research Institute, Dublin Institute of

Technology, Kevin Street, Dublin 8, Ireland. Fax: + 003531402

7901; Phone: 00353 (0)1 402 7982; E-mail: fuormg.tian@dit.ie
Although the SERS effect in metal nanoparticles is proposed to derive from the local fields associated with the excitation of surface plasmon resonances by the Raman source, nanospheres suffer from low enhancement levels that vary widely from particle-to-particle and fluctuate with their environment 5 . In fact, more recent explanations of SERS on metal nanoparticles are based not on intrinsic nanoparticle surface plasmons, but local field "hotspots" 6 due to surface roughness ${ }^{7}$, between aggregated metallic NPs ${ }^{8}$ or between nanoparticles ${ }^{9}$ and a metal surface ${ }^{10}$ and that the SERS contribution of such hotspots can dominate the observed response ${ }^{11}$. An alternative way to increase the local electromagnetic field associated with SPR is to increase, in a systematic fashion, the local curvature of nanomaterials. A 10-100 times higher field strength was estimated at the vertices of silver nanotriangles compared to the surface of nanospheres ${ }^{12}$. Recently, a new class of star-shaped gold nanoparticle with sharp edges and tips, referred to as nanostars, has been shown to exhibit a very high sensitivity to local changes in the dielectric environment, as well as larger enhancements of the electric field around the nanoparticles ${ }^{13}$. Similar results have been found for other nanoparticles with sharp features ${ }^{4,14}$.

The methods to produce gold nanospheres, nanotriangles and nanostars with a high degree of control of the size/shape distribution can be considered as routine, and thus a direct comparison of the SERS efficiency of the different structures is warranted. This study will detail the synthesis and UV/visible absorption of such nanoparticles, and will explore their relative efficiencies for SERS 
using the organic dye molecule rhodamine $6 \mathrm{G}(\mathrm{R} 6 \mathrm{G})$ as a probe, in an attempt to elucidate routes towards optimised SERS probes. Aggregation of nanospheres is induced to demonstrate the further enhancement due to interparticle local field "hotspots" and to compare with the intrinsic enhancement induced at the vertices of nanotriangles and nanostars.

\section{Experimental}

$\mathrm{HAuCl}_{4} \mathrm{H}_{2} \mathrm{O}, \quad \mathrm{NaBH}_{4}, \quad$ ascorbic acid, $\mathrm{AgNO}_{3}$, cetyltrimethylammonium bromide (CTAB), and $10 \mathrm{~nm}$ gold colloid suspensions $\left(6 \times 10^{12} / \mathrm{mL}\right)$ were purchased from Sigma Aldrich (Dublin, Ireland). Ultrapure deionized water (resistivity greater than

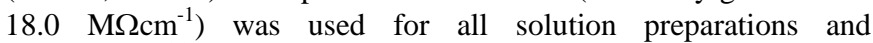
experiments ${ }^{15}$.

Gold nanospheres, with diameter $\sim 150 \mathrm{~nm}$, were prepared using the seed-mediated growth method ${ }^{15}$. $0.01 \mathrm{~mL}$ of the $10 \mathrm{~nm}$ gold colloid seed suspension (Sigma Aldrich) was added to $10 \mathrm{~mL}$ of the reducing solution, containing trisodium citrate and ascorbic acid, under vigorous stirring over a time of $\sim 45 \mathrm{~min}$. Immediately after the addition was complete, the mixture was brought to the boil and maintained at this temperature for $\sim 30 \mathrm{~min}$. In order to evaluate the effect of aggregation on SERS from the gold nanospheres, acidic conditions were employed to induce nanoparticle aggregation ${ }^{16}$. $\mathrm{HCl}$ at a concentration of $1 \mathrm{M}$ was added dropwise to aqueous suspensions of $150 \mathrm{~nm}$ nanospheres and the $\mathrm{pH}$ value was adjusted between 7 to 4 . At $\mathrm{pH}=4$, the particle solution was seen to undergo a colour change indicative of aggregation ${ }^{17}$.

Gold nanotriangles were prepared by a reduction of chloroauric acid $\left(\mathrm{HAuCl}_{4}\right)$ using sodium thiosulfate as reducing agent ${ }^{1,18}$. The synthesis requires aqueous ascorbic acid $(75 \mathrm{~mL}, 10 \mathrm{mM})$, and 0.01 $\mathrm{mL}$ of the $10 \mathrm{~nm}$ gold colloid suspension (Sigma Aldrich) in $5 \mathrm{~mL}$ distilled water, to which $3 \mathrm{~mL}$ of $0.5 \mathrm{mM} \mathrm{AgNO}$ is added at a rate of $1 \mathrm{~mL} / \mathrm{min}$. $150 \mu \mathrm{L}$ of $10 \mathrm{mM}$ ascorbic acid solution and $0.5 \mathrm{mM}$ $\mathrm{HAuCl}_{4}$ are then added at $0.2 \mathrm{~mL} / \mathrm{min}$ while stirring vigorously. Within the initial $5 \mathrm{~min}$ of the reaction, the colour of the solution changed from yellow (gold salt) to brownish. This change of colour indicated the formation of GNPs. The reaction was stopped after 15 minutes, to limit the particle size to $\sim 120 \mathrm{~nm}$ and prevent aggregation, by centrifugation of the nanoparticles ${ }^{19}$. Nanotriangles were subsequently washed by deionized water three times under centrifugation at 4500 RPM for 15 mins.

Gold nanostars were prepared in aqueous phase via the surfactantdirected, seed-mediated growth method as described in the literature 20. Growth solution was prepared by adding $0.20 \mathrm{~mL}$ of $0.01 \mathrm{M}$ $\mathrm{HAuCl}_{4}: 4 \mathrm{H} 2 \mathrm{O}$ to $4.5 \mathrm{ml}$ of $0.1 \mathrm{M} \mathrm{CTAB}$ in a plastic test tube while gently mixing. To this solution, $0.030 \mathrm{~mL}$ of $0.01 \mathrm{M} \mathrm{AgNO}_{3}$ was added. After mixing, the colour of the solution becomes brownish yellow. Then, $0.032 \mathrm{~mL}$ of $0.1 \mathrm{M}$ ascorbic acid was added, resulting in a colourless solution. Finally, $0.01 \mathrm{~mL}$ of the $10 \mathrm{~nm}$ gold colloid suspension (Sigma Aldrich) was added. After gentle mixing, the solution was kept in a water bath at room temperature. The eventual blue-purple colour of the growth solution indicates gold nanostar formation ${ }^{20}$. The reaction was stopped after 30 minutes, to limit the particle size to $\sim 150 \mathrm{~nm}$ and prevent aggregation ${ }^{21}$. Khoury et al. have demonstrated that prolonged reaction time can result in increased size of nanostars ${ }^{21}$. Nanostars were subsequently washed by deionized water three times under centrifugation at 4500 RPM for 15 mins. The surfactant CTAB and ascorbic acid were removed by washing with water. Once the reaction was stopped and the products remained stable over 6 months.
A Perkin Elmer Lambda 900 UV/VIS/NIR Spectrometer and Zetasizer Nano ZS analyser (Malvern Instruments, Worcestershire, UK) were used to measure the absorbance, hydrodynamic particle size and zeta potentials of the nanoparticles and to observe the formation and/or aggregation of NPs.

In parallel, the three different geometry nanoparticles (spheres, triangles, star) as well as nanosphere aggregates, at a nanoparticle number concentration $(\mathrm{Nn})$ of $3 \times 10^{3}$ particle/mL, were deposited on a 300-mesh grid for TEM (Ted Pella Formvar/Carbon type B) by drop casting $10 \mu \mathrm{L}$ of the aqueous solution of nanoparticles. Similar nanoparticle solutions were dropped onto prewashed silicon substrates and spin coated at a speed of $1000 \mathrm{rpm}$ for 20 seconds for SEM. The samples were dried in air and characterised by Electron Microscopy using a Hitachi SU6600 FESEM instrument at an acceleration voltage of $25 \mathrm{kV}$. Scanning EM images were taken using the SE detector and the Scanning Transmission EM images were taken using the TE detector.

Raman spectroscopy was performed with a HORIBA Jobin Yvon HR800 spectrometer with either a $50 \mathrm{~mW} 532 \mathrm{~nm}$ or a $300 \mathrm{~mW} 785$ $\mathrm{nm}$ diode laser as source. Spectral data was collected using a $10 \times$ microscope objective over the range $400-1800 \mathrm{~cm}^{-1}$ with a $10 \mathrm{sec}$ integration time. The detector used was a 16-bit dynamic range Peltier cooled CCD detector ${ }^{22,23}$.

SERS samples were prepared by mixing $25 \mu$ of GNP solution with $25 \mu \mathrm{l}$ of aqueous solutions of the probe molecule (R6G) at varying concentrations. In all cases, the nanoparticle number was estimated based on the concentrations of initial seed nanospheres. In the case of the aggregated nanospheres, nanoparticle numbers quoted for all experiments are those of the initial, unaggregated suspensions. Final nanoparticle concentrations $(\mathrm{Nn})$ of $3 \times 10^{3}, 3 \times 10^{6}$ or $3 \times 10^{9} / \mathrm{mL}$ were employed for the range of experiments. For the probe molecule, concentrations were varied over the ranges $0.1-10 \mu \mathrm{M}$ (R6G), for each nanoparticle concentration. SERS effects in R6G have been well studied and, for example, it has been shown that silver can cause SERS on R6G in the $\mu \mathrm{M}$ range ${ }^{24}$. Solutions were dropped onto $\mathrm{CaFl}_{2}$ substrates and measured immediately. For comparison, spectra of the probe molecule R6G alone were recorded from $1 \mathrm{M}$ aqueous solutions.

\section{Results}

\subsection{Particle Sizing and Zeta Potential}

Table 1 presents the key physicochemical characteristics within each group of nanoparticles used in the present study, as determined by a combination of DLS, Zeta potential, electron microscopy and absorption spectroscopy. In suspension, the DLS of the Gold nanosphere solutions indicates a monomodal dispersion with a hydrodynamic diameter of $150 \pm 9 \mathrm{~nm}$ and a zeta potential of $16.5 \pm 0.4 \mathrm{mV}$. In acidic conditions, the particle size distribution dramatically increases to $1030 \pm 37 \mathrm{~nm}$, while the zeta potential is changed to $-7.8 \pm 0.2 \mathrm{mV}$ (Table 1 ). The acid reduces the absolute value of the negative zeta potential although it remains negative even at $\mathrm{pH}=4$. Zeta potential is varied most commonly by $\mathrm{pH}$ adjustment 21

Gold nanotriangles and nanostars similarly show a monomodal suspension with hydrodynamic diameters of $135 \pm 13 \mathrm{~nm}$ and $148 \pm 11$ $\mathrm{nm}$ respectively, although it should be noted that the DLS algorithm for diameter calculation assumes spherical particles. The measured zeta potentials were $-21.6 \pm 1.9 \mathrm{mV}$ and $29.6 \pm 3.6 \mathrm{mV}$ respectively. 
Although they have been washed rigorously, the positive zeta potential of the nanostars, in contrast to the negative values observed for both other nanoparticles, is most likely due to residual cationic $\mathrm{CTAB}$ which is known to form bilayer structures on the surface of metals, resulting in a positive zeta potential ${ }^{20}$. However, it has been demonstrated that the shape of nanoparticles plays the dominant role in determining surface enhancement. Cube-like CTAB-capped gold nanoparticles were shown to provide 4 times higher SERS from human immunoglobulin $\mathrm{G}$ than spherical CTAB-capped gold nanoparticles ${ }^{25}$.

Table 1. Physico-chemical characteristics of each different GNP type

\begin{tabular}{llllll}
\hline Samples & $\begin{array}{l}\text { Hydrodyn } \\
\text { amic } \\
\begin{array}{l}\text { Diameter } \\
(\mathrm{nm})\end{array}\end{array}$ & $\begin{array}{l}\text { Zeta } \\
\text { Potential } \\
(\mathrm{mv})\end{array}$ & $\begin{array}{l}\text { Number } \\
\text { of vertices }\end{array}$ & $\begin{array}{l}\text { Surface } \\
\text { area } \\
\left(\mathrm{nm}^{2}\right) \\
\text { per NP }\end{array}$ & $\begin{array}{l}\lambda_{\max } \\
\mathrm{nm}\end{array}$ \\
\hline Nanosphere & $150 \pm 9$ & $-16.5 \pm 0.4$ & - & 70650 & 560 \\
& $1030 \pm 37$ & $-7.8 \pm 0.2$ & - & 10,158 & 660 \\
$\begin{array}{l}\text { Nanosphere } \\
+\mathrm{HCl}\end{array}$ & $135 \pm 13$ & $-21.6 \pm 1.9$ & 3 & 62353 & 800 \\
Nanotriangle & & & & & \\
& $148 \pm 11$ & $29.6 \pm 3.6$ & 6 & 24335 & $600 /$ \\
Nanostar & & & & & 785 \\
& & & & & \\
\hline
\end{tabular}

\subsection{Electron Microscopy}

Electron microscopy was employed to confirm that the change in the DLS profile of the nanospheres in acidic conditions was due to aggregation, and to visualise the nanotriangles and nanostars in their isolated form. Figure 1 shows electron microscopy images of 150 $\mathrm{nm}$ gold nanospheres, precipitated from neutral (Figure 1a) and $\mathrm{pH} 4$ (figure 1b-e) aqueous solutions. In the aggregated form, although isolated spheres are still evident, the bulk of the nanospheres exist as dimers, trimers or higher order aggregates. This is consistent with the observed dramatic increase in hydrodynamic diameter and lower zeta potential (Table 1). $\mathrm{HCl}$ decreases the absolute value of the zeta potential, resulting in a decreased repulsion between the NPs and consequent aggregation ${ }^{16}$.

As shown in Figure $2(\mathrm{a}-\mathrm{c})$, nanotriangles, precipitated from aqueous solution of neutral $\mathrm{pH}$, are flat regular structures with three congruent edge lengths in the $100 \mathrm{~nm}$ to $120 \mathrm{~nm}$ range. Typically, each tip is $\sim 60$ degrees (Fig. 2 a-c).

Nanostars typically have a central core and 6 vertices in a 3 dimensional arrangement. The length of the vertices is averaged at $35 \mathrm{~nm}$ and the angle at the vertex is less than 30 degrees (Fig. 2, d-f). Based on idealised geometric structures, nanospheres had the largest surface area per particle, followed by nanotriangles, while the nanostars had the smallest estimated surface area (Table 1). If a perfect spherical geometry is assumed, a hydrodynamic diameter of $1030 \mathrm{~nm}$ for nanosphere aggregates yields a surface area of $3.3 \times 10^{6}$ $\mathrm{nm}^{2}$. Rationing the volumes of the nanosphere aggregates and nanospheres gives an estimate of 325 nanospheres per aggregate, and therefore a surface area per nanoparticle of $\sim 1 \times 10^{4} \mathrm{~nm}^{2}$ per nanosphere in an aggregate, less than that of the unaggregated nanospheres.
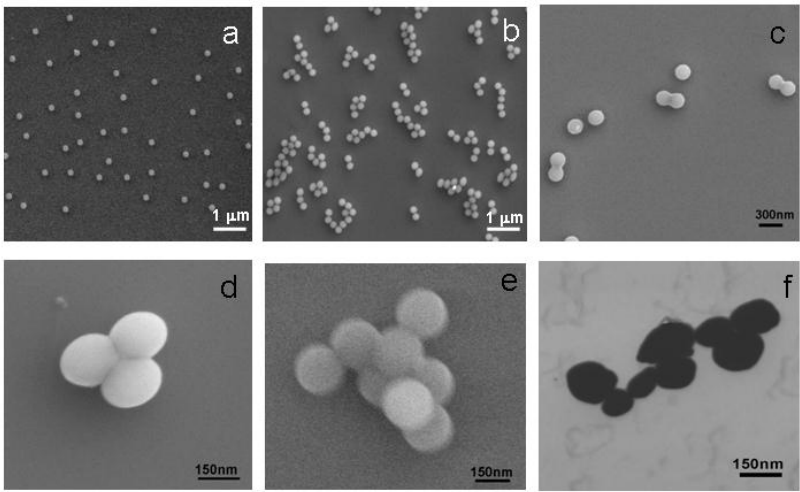

Figure 1: (a) SEM image of nanospheres, (b-e) SEM images of aggregated nanospheres: (f) shows an STEM image of aggregates of the same nanospheres.
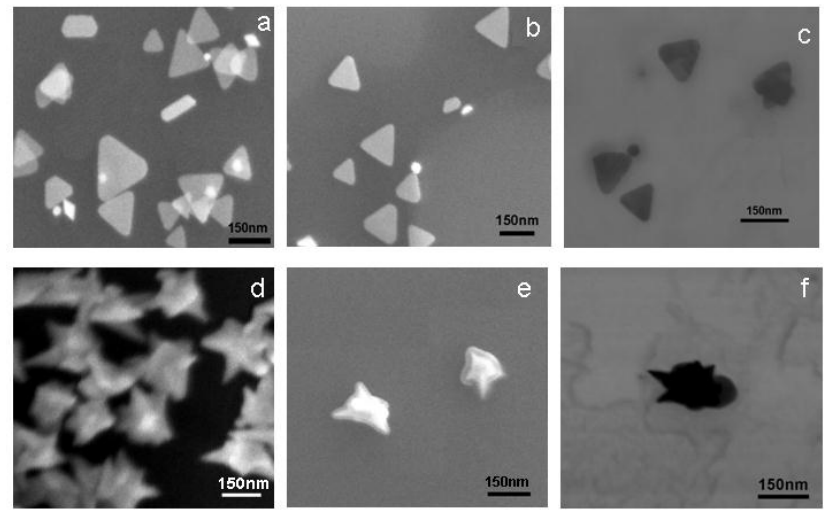

Figure 2: Electron microscopy images of nanotriangles (a-c) and nanostars (d-f). Images of (a), (b), (d) and (e) are taken by SEM. Images of (c) and (f) are STEM images.

\subsection{UV-Vis-NIR absorption spectroscopy}

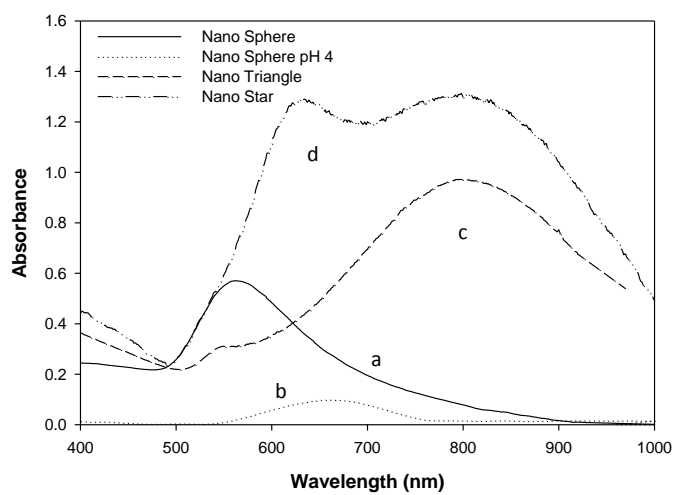

Figure 3: UV-vis absorption spectra of (a) nanospheres, (c) nanotriangles and (d) nanostars in aqueous solutions at a concentration of $\mathrm{N}_{\mathrm{n}}$ of $6 \times 10^{8}$ particles $/ \mathrm{ml}$. The dotted line in (b) illustrates the spectrum of the nanospheres in aqueous solution at $\mathrm{pH}$ 4. 
Figure 3 shows the UV/vis/NIR absorption spectra of aqueous suspensions of the gold nanospheres, before (a - solid line) and after (b - dotted line) aggregation. Before aggregation, the spectral profile shows the SPR at $\lambda \max \sim 560 \mathrm{~nm}$, typical of gold nanospheres ${ }^{26}$. Upon the addition of $\mathrm{HCl}$ to the nanosphere solution, a colour change from red to blue-grey was immediately observable. The absorbance is dramatically reduced and the SPR band is red shifted to $\lambda \max \sim 660 \mathrm{~nm}$. The observed behaviour is consistent with that previously reported for aggregation of gold nanospheres ${ }^{26}$. Notably, whereas the optimum wavelength for excitation of the SPR for monodisperse gold nanospheres is $\sim 560 \mathrm{~nm}^{27}$, that for aggregated nanospheres is substantially longer ${ }^{26}$.

The spectrum of the nanotriangles shows a single SPR band at $\sim 800$ $\mathrm{nm}$ (Fig. $3 \mathrm{c}-$ dashed line). This feature corresponds to the in-plane dipolar mode, which falls in the near infrared range ${ }^{28}$. The positioning of the SPR maximum shifts to longer wavelength, from $\sim 800 \mathrm{~nm}$ to $\sim 950 \mathrm{~nm}$, with reaction time, as a result of increasing particle size ${ }^{19}$. The nanostars show an absorbance spectrum which is doubly peaked, at $\sim 600 \mathrm{~nm}$ and $\sim 800 \mathrm{~nm}$ (Fig. $3 \mathrm{~d}-$ dotted/dashed line). These two peaks derive from the two constituent structures of the nanostar, the spherical core and the peripheral vertices. The spherical core results in a SPR similar to the unaggregated nanospheres (Fig. 1a) while the multiple vertices contribute SPR in the near infrared range (Fig. 1d) ${ }^{13}$. The positioning of the second SPR peak increases from $\sim 785 \mathrm{~nm}$ to $\sim 900 \mathrm{~nm}$, with reaction time, as a result of increasing particle size ${ }^{21}$.

The optical properties of the GNPs in suspension thus reflect the characteristics of the local fields associated with the SPR. While the nanospheres have an intrinsic SPR at $560 \mathrm{~nm}$, their aggregation, associated with the generation of local field hotspots between the spheres, causes a redshift of the SPR. In the case of the nanotriangles and the nanostars, the sharp vertices similarly act as local field hotspots resulting in SPRs shifted to the near infrared region of the spectrum $^{28,29}$.

\subsection{Raman Spectroscopy}

As shown in Figure 4, Raman scattering was not observable under the measurement conditions employed in the presence of monodisperse gold nanospheres at a source wavelength of either 532 $\mathrm{nm}$ or $785 \mathrm{~nm}$. At a $\mathrm{pH}=4$, however, although no significant Raman scattering was observable using $532 \mathrm{~nm}$ as source, the Raman signal was significantly enhanced at $785 \mathrm{~nm}$. Although $532 \mathrm{~nm}$ is close to the SPR band of the monodisperse nanospheres, no SERS effect is observed, consistent with the requirement for aggregation to generate local hotspots at which the local field is substantially enhanced, resulting in a shift of the SPR band to $\sim 660 \mathrm{~nm}$. In the aggregated suspensions, the particles are no longer resonant at 560 $\mathrm{nm}$ (Fig. 3a) and no SERS effect is observable using $532 \mathrm{~nm}$ as source, but a strong SERS signal is observable using $785 \mathrm{~nm}$, as a result of the red shifted SPR.

In the absence of aggregation, the $785 \mathrm{~nm}$ laser is resonant with the primary SPR bands of both the nanostars and nanotriangles. These isotropic nanoparticles have strong SPR throughout the visible and near-IR (NIR) regions of the spectrum ${ }^{29}$. Figure 5a compares the Raman spectrum at $785 \mathrm{~nm}$ of the $1 \mathrm{M}$ solution of $\mathrm{R} 6 \mathrm{G}$ with the SERS spectrum of $5 \mu \mathrm{M}$ R6G in a suspension of gold nanostars.

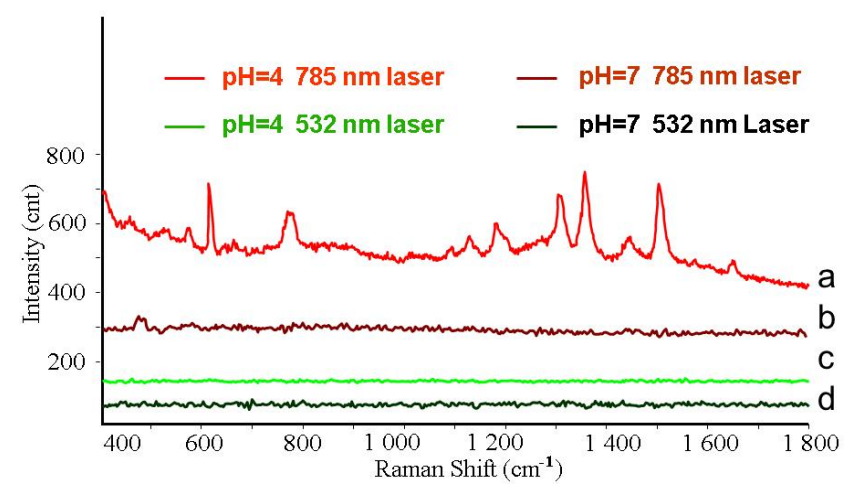

Figure 4: Raman spectra of gold nanosphere and $10 \mu \mathrm{M}$ R6G using $532 \mathrm{~nm}$ and $785 \mathrm{~nm}$ as source. The red line shows molecules with nanospheres at $785 \mathrm{~nm}$ and $\mathrm{pH}=7$ (a), the maroon line shows molecules with nanospheres at $785 \mathrm{~nm}$ and $\mathrm{pH}=4$, the green line shows the spectra of molecules with nanospheres at $532 \mathrm{~nm}$ and $\mathrm{pH}=7$ (c), the dark green shows the spectra of molecules with nanospheres at $532 \mathrm{~nm}$ and $\mathrm{pH}=4(\mathrm{~d})$. The particle concentration is 3 x $10^{9} / \mathrm{ml}$. The spectra are offset for clarity.
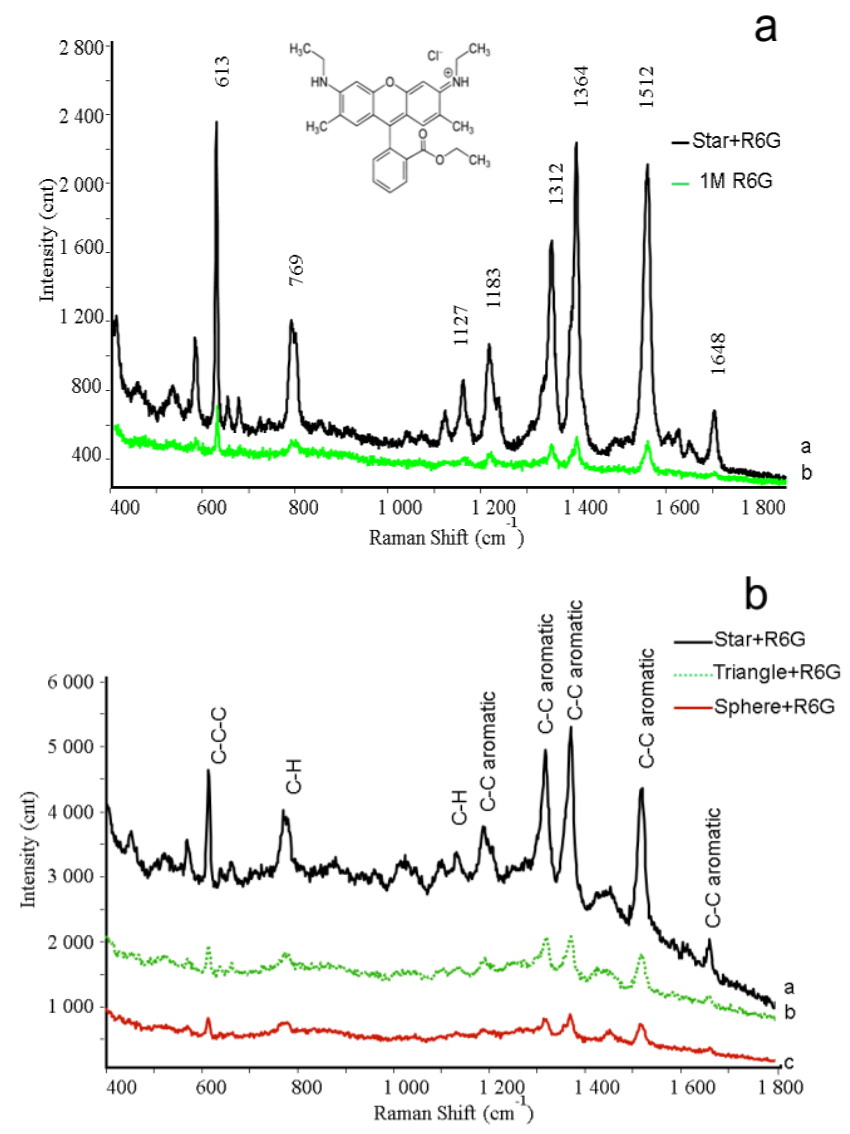

Figure 5: SERS spectra of R6G with aggregated-nanospheres, nanotriangles and stars at a concentration $3 \times 10^{9}$ particles $/ \mathrm{ml}$. (a) comparison of the SERS spectrum of $5 \mu \mathrm{M}$ R6G in gold nanostar solution (a, dark), the Raman spectrum of 1 M R6G alone (b, green), (b) Comparison of $5 \mu \mathrm{M}$ R6G SERS spectrum in solutions of gold nanostars (a, dark) nanotriangles (b, green dash) and aggregated nanospheres (c, red). 
Three different groups of modes were observable for R6G, associated with $\mathrm{C}-\mathrm{C}-\mathrm{C}$ ring in-plane bending at $613 \mathrm{~cm}^{-1}$, out-ofplane bending at $769 \mathrm{~cm}^{-1}$ and ring breathing (RB), such as aromatic C $-\mathrm{C}$ stretching at $1183,1312,1364,1512 \mathrm{~cm}^{-1}$ and $1648 \mathrm{~cm}^{-1}, v(\mathrm{C}-$ $\mathrm{H})$ at $1127 \mathrm{~cm}^{-1}$. The vibrational modes observed in the SERS spectra (Fig. 5b) are assigned to the corresponding vibrational modes, consistent with the observations of SERS of R6G on silver nanospheres ${ }^{30}$. Consistent with literature ${ }^{30,31}$, eight Raman bands with strong scattering intensities are observed at 613, 775, 1130, $1278,1364,1389,1512$, and $1651 \mathrm{~cm}^{-1}$. While all these modes were strongly enhanced on nanostars in the SERS measurements, only a few were detectable in the bulk Raman spectra of R6G. A comparison of SERS and Raman spectra of the molecule shows only small shifts of these modes, making it difficult to determine the adsorption site of the molecule on the GNP surface. The results suggest that R6G and GNPs do not interact strongly. On the other hand, the strong enhancements observed for all groups of modes mentioned previously suggests the central carbon atom, nitrogen atoms, and $\pi$ electrons in the phenyl ring as possible interaction sites 24 .

Significantly, in all measurements made, as shown in Figure 5b, the magnitude of the SERS response was highest for the gold nanostars, intermediate for the nanotriangles, lower for the aggregated nanospheres, and negligible for the unaggregated nanospheres (Fig. 3 ). The results are consistent with the requirement for enhanced electric fields at hotspots associated with aggregated nanospheres, or the vertices of triangular or star shaped nanoparticles.
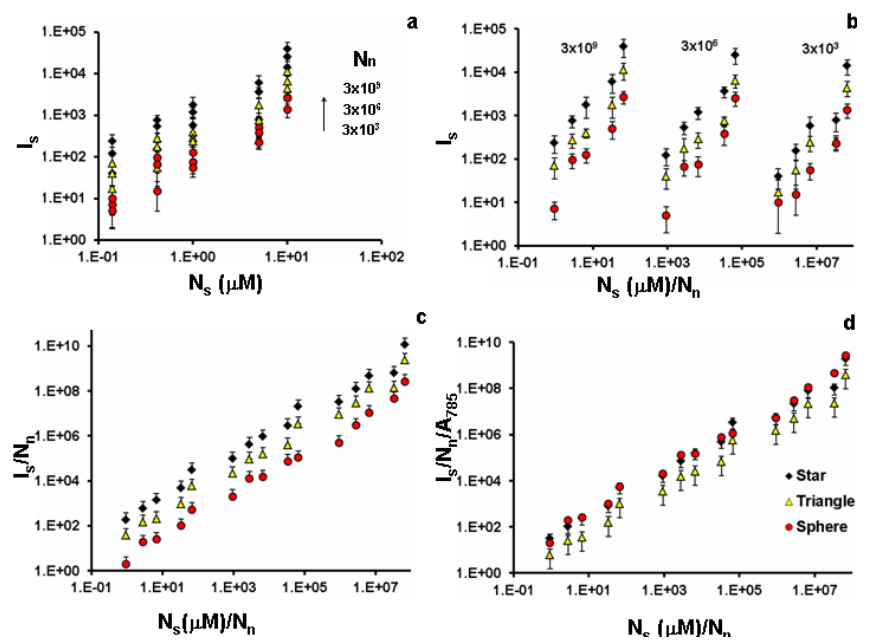

Figure 6: The intensity of the SERS peaks (above baseline) $\left(\mathrm{I}_{S}\right.$ arbitrary units) of R6G $\left(1364 \mathrm{~cm}^{-1}\right)$ at different concentrations of R6G $\left(N_{S}\right.$, in $\left.\mu \mathrm{M}\right)$ for each nanoparticle type (in each plot, Blue diamond $=$ NanoStar, Yellow triangle $=$ NanoTriangle, Red disc $=$ aggregated Nanosphere) and each Nanoparticle number $\left(\mathrm{N}_{\mathrm{n}}\right.$, per $\left.\mathrm{mL}\right)$ range. (a) $I_{S}$ vs $N_{S}$, (b) $I_{S}$ vs $N_{S} / N_{n}$, (c) $I_{S} / N_{n}$ vs $N_{S} / N_{n}$ (d) $I_{S} / N_{n}$ vs $N_{S} / N_{n}$ normalised to absorbance at $785 \mathrm{~nm}$, A785.

Figure 6a plots the Raman intensity $\left(\mathrm{I}_{\mathrm{S}}\right)$ of the $1364 \mathrm{~cm}^{-1}$ mode versus the dye concentration $\left(\mathrm{N}_{\mathrm{S}}\right)$ for each nanoparticle concentration. For each nanoparticle type, there is a significant difference between the SERS intensity observed for a fixed nanoparticle concentration, and that signal is increased with the number of nanoparticles present at a given dye concentration. Note that the plot is $\log / \log$ and the superlinear order of the slope for each curve $(\sim 1.2)$ indicates that the enhancement factor (normally defined as $E F=I_{S} N_{R} / I_{R} N_{S}$, where $I_{S}$ and $I_{R}$ are, respectively, the SERS and normal Raman intensities that result from sampling a concentration of $N_{S}$ and $N_{R}$ molecules ${ }^{21}$ ) is not independent of concentration of the dye or nanoparticles and the relationship between the Raman signal per dye molecule and the number of nanoparticles in solution is not immediately apparent.

The datasets are further separated when considering the Raman signal as a function of dye molecules per nanoparticle, as shown in Figure 6 b. For each nanoparticle type, a similar SERS intensity is observed for substantially different regimes of the parameter $I_{s} / N_{n}$, depending on the concentration of nanoparticles. However, when considering the SERS intensity per nanoparticle, as a function of the number of R6G molecules per nanoparticle, a more continuous behaviour is observed for each nanoparticle type, over the concentration range studied, as shown in Figure 6c.

In Figure 6c, a clear dependence of the SERS signal, and therefore enhancement factor, EF, on nanoparticle shape is observable. Although it is not accentuated in the $\log / \log$ format of the graph, there remains a significant difference between the SERS per nanoparticle at a given concentration of dye per nanoparticle, in the sequence (aggregated) nanosphere < nanotriangle < nanostar.

\subsection{Number of hotspots per particle}

Given that the unaggregated nanospheres gave negligible SERS signals under these experimental conditions, the results clearly indicate that the number of intrinsic "hotspots" per particle (and for equal $\mathrm{Nn}$, per unit volume) is a primary determinant on the SERS signal observable. This increases as nanospheres <nanotriangles $<$ nanostars. Although it cannot be assumed that all "hotspots" are of equal strength, also implicit in the results is that the average number of hotspots per nanoparticle in aggregated nanospheres is significantly less than that in either nanotriangles or nanostars. This is also evident in both the progressive red shift and increase in absorbance of the SPR with increasing local field associated with hotspots, observed in Figure 3. It should be noted, however, that in a study of the size dependence of nanostars by Khoury et al., the dependence of absorption strength and wavelength positioning of the SPR is not monotonic, and that with increasing reaction time, although the strength of the SPR continues to increase, the wavelength positioning ceases to redshift, and even begins to blue shift.

An interesting observation is made when the $\mathrm{I}_{\mathrm{S}} / \mathrm{Nn}$ signals of Figure $6 \mathrm{c}$ are normalised by the relative absorbance of each nanoparticle type, nanostar: nanotriangle: aggregated nanosphers, at the Raman excitation wavelength $(15: 10: 1)$. As shown in Figure $6 \mathrm{~d}$, the normalised SERS signals overlap for all nanoparticle types, over the full range of concentrations of normalised dye concentrations. Therefore, despite the range of responses indicated for the different nanoparticle types and concentration ranges indicated in Figure 6a-c, the SERS responses can be mapped on to a single behaviour. Notably, the superlinear behaviour of Figure 6a has reduced to a slightly sublinear behaviour (slope $=0.93$ ) for all nanoparticle types.

\section{Discussion}

The fundamental process of SERS is based on the local field enhancement in the region of metallic nanostructures upon excitation of the SPR ${ }^{6,32}$. Gold nanoparticles and nanostructured substrates thus represent an ideal candidate for sensitive SERS detection and imaging in the visible region, as their SPR resonance occurs at $560 \mathrm{~nm}{ }^{33}$. However, increasingly, reports of optimised SERS 
processes using gold nanoparticles and nanostructured substrates utilise longer source wavelengths of $633 \mathrm{~nm}$ or $785 \mathrm{~nm}^{5,34}$, indicating that it is not the intrinsic SPR of the gold nanoparticles which gives rise to the strong SERS effect.

The measurements presented here confirm that the SERS effect due to the SPR of isolated nanospheres (at 532nm) is negligible compared to that of nanosphere aggregates (at $785 \mathrm{~nm}$ ). In solution, as confirmed by DLS and TEM, the nanospheres spontaneously aggregate upon the addition of $\mathrm{HCl}$, leading to a colour change from orange/red to blue-grey, as the SPR shifts from $560 \mathrm{~nm}$ to $\sim 660 \mathrm{~nm}$. Such junctions give rise to local field "hotspots" which are the source of the SERS effect in many studies, and are typically excited by source wavelengths of $>600 \mathrm{~nm}$. For example, Drescher et al. have demonstrated that the acidic environment of endosomes causes nanoparticle aggregation into dimers and trimers leading to an increased SERS effect at $785 \mathrm{~nm}{ }^{16}$. Bonifacio et al. have demonstrated that negligible SERS effects are observable in human serum samples, in which the serum proteins form a protein corona ${ }^{33}$, ${ }^{34}$, which coats the nanoparticles and prevents them from aggregating and forming hotspots, whereas, once the proteins are filtered out, strong and reproducible SERS signals can be recorded, again using $785 \mathrm{~nm}$ as source ${ }^{35}$.

Thus, aggregation of nanoparticle provides hotspots which result in significantly higher SERS effects and the optimum source wavelength is considerably shifted from that of the SPR of the intrinsic nanoparticle. Braun et al. demonstrated that controlled aggregation can produce significant increases in the SERS response from silver nanoparticles ${ }^{35}$. Wustholtz et al. have explored structureproperty relationships governing the SPR and SERS effects in gold nanosphere aggregates ${ }^{36}$ and Laurence et al. have demonstrated good correlations of enhancement factors with degree of aggregation in encapsulated silver nanoaggregates ${ }^{37}$. The SERS response from substrates of hexagonally packed silver nanodiscs and nanorods has been demonstrated to vary over four orders of magnitude, dependent on the spacing ${ }^{6}$. However, aggregation is not a well controlled phenomenon and adds further uncertainty and variability to an already complex system. An alternative way to increase the local electromagnetic field associated with the SPR is to increase the local curvature of nanomaterials. It has been shown that when two spherical nanoparticles are aggregated or close enough, the SPR band is split into two components: longitudinal (low frequency) and transverse (high frequency) ${ }^{38}$. In spherical particles, these two modes (quadrupole and dipole) are not distinguishable from one another ${ }^{39}$. In the case of nanotriangles, due to their anisotropic shape, four different plasmon resonances have been observed: inplane dipole, quadrupole, out-of-plane dipole, and quadrupole $e^{40}$. Nanostars contain a higher number of sharp corners and edges, and they have their own unique character as more complex anisotropically shaped nanoparticles and the modes oscillate at markedly different frequencies in both $\mathrm{Au}$ and $\mathrm{Ag}$ materials ${ }^{41,} 42$. Roughly, these modes originate from the degree and direction of polarization of the electron cloud relative to the incident electric field $^{43,44}$.

Early theoretical simulations indicated that the local electric field enhancement in metal nanostructures is strongly dependent on the shape of the metal protrusion, both through the effectiveness of the so-called "lighting rod mechanism" and through the shape dependence of the SPR frequency ${ }^{45,46}$. The lightning rod effect can result in the largest electric field near the sharpest surface, e.g., at the sharp ends of nanoparticles ${ }^{47}$. Nanotriangles contain three sharp vertices or "tips" of $\sim 60$ degrees that contribute significantly to their optical and electronic properties, although in practice, mixtures with varying degrees of tip truncation and rounding can be found. Nanostars contain $\sim 5$ or more vertices of angles $\sim 30$ degrees resulting in considerably higher local field enhancement and consequently higher SERS, per nanoparticle.

Much effort has been devoted to establishing a correlation between SERS and the absorbance of nanomaterials. Talley el al found aggregated nanospheres and dimers have higher absorbances at 700 and $785 \mathrm{~nm}$ respectively giving rise to higher SERS efficiencies ${ }^{48}$. Wustholz, K. L. et al also reported that SERS efficiency for aggregated nanospheres is related to the positioning of the SPR between $600-900 \mathrm{~nm}^{36}$. Similar work has been published recently comparing SERS from arrays of gold nanodisc with varied diameter and interdisc spacing ${ }^{26}$. In this study, the absorbance spectra of Figure 3 give a clear indication of the relationship between the nanoparticle absorbances and the nanoparticle structures. Notably, however, Khoury et al. have demonstrated that for nanostars monitored under controlled growth conditions the redshifting of the SPR resonance with reaction time is not monotonic ${ }^{21}$. The empirical observation of Figure $6 c$, that, when normalised for the nanoparticle absorbance at the Raman source wavelength, the SERS per nanoparticle overlaps for each nanoparticle type, indicates that this simple experimental parameter can be used as a guide to optimising nanoparticle synthesis and experimental design.

\section{Conclusions}

The study clearly demonstrates that the SERS signal due to the excitation of the SPR of isolated nanospheres is negligible compared to that of their aggregates, which requires longer wavelength excitation, in this instance at $785 \mathrm{~nm}$. Aggregation gives rise to local field hotspots which significantly enhance the local field, and red shift the SPR. A similar result, without the requirement of aggregation, can be achieved by increasing the local curvature of the nanoparticle surface, as is the case for nanotriangles and nanostars. The SERS effect for the common organic dye R6G is observed to systematically increase in the sequence nanospheres < nanosphere aggregates < nanotriangles < nanostars, as a result of the increased number and strength of local field hotspots.

The results presented here compare the SERS efficiencies of gold nanoparticles of differing shapes, but equivalent dimensions. The SERS intensity is seen to be well correlated with the optical absorption and indeed, when normalised to the absorbance at the Raman sources wavelength, the SERS intensity per nanoparticle, is seen to be equivalent for all nanoparticle types, indicating that optimisation of the SERS response can be achieved by optimising the absorbance of the nanoparticle at the Raman source wavelength.

\section{Acknowledgement}

F.B, A.C, and H.J.B acknowledge the support of Science Foundation Ireland 11/PI/1108. F.T. is funded under a Marie Curie Intra European Fellowship, Grant Agreement n PIEF-GA-2012-332462.

\section{References}

1. C. Bao, N. Beziere, P. del Pino, B. Pelaz, G. Estrada, F. Tian, V. Ntziachristos, J. M. de la Fuente and D. Cui, Small, 2013, 9, 68-74.

2. J. B. Jackson and N. J. Halas, Proc Natl Acad Sci U S A, 2004, 101, 17930-17935. 
3. S. Bhaskar, F. Tian, T. Stoeger, W. Kreyling, J. M. de la Fuente, V. Grazu, P. Borm, G. Estrada, V. Ntziachristos and D. Razansky, Part Fibre Toxicol, 2010, 7, 3.

4. Bin Ren, Gennaro Picardi and B. Pettinger, Review of Scientific Instruments, 2004, 75, 837-841.

$5 . \quad$ J. L. J. PérezI, J. F. S. RamírezI, R. G. FuentesI, A. CruzOrealI and J. L. H. Pérez, Brazilian Journal of Physics, 2006, 36

6. M. Moskovits, hys Chem Chem Phys 2013, 15, 53015311.

7. P. K. Aravind and H. Metiu, Surf Sci 1983, 124, 506-528.

8. K. Kneipp, H. Kneipp, I. Itzkan, R. R. Dasari and M. S. Feld, J. Phys.: Condens. Matter 2002, 14, R597-R624.

9. P. K. Aravind and H. Metiu, Chemical Physics Letters 1980, 74, 301-305.

10. P. K. Aravind, A. Nitzan and H. Metiu, Surf Sci 1981, 110, 189-204.

11. Y. Fang, N. H. Seong and D. D. Dlott, Science 2008, 321, 388-391.

12. V. S. Tiwari, T. Oleg, G. K. Darbha, W. Hardy, J. P. Singh and Paresh Chandra Ray, Chemical Physics Letters, 2007, 446, 77-82.

13. F. Hao, C. L. Nehl, J. H. Hafner and P. Nordlander, Nano Lett, 2007, 7, 729-732.

14. C. L. Nehl, H. Liao and J. H. Hafner, Nano Lett, 2006, 6, 683-688.

15. C. Ziegler and A. Eychmüller, J. Phys. Chem. C. , 2011, 115, 4502-4506.

16. D. Drescher, P. Guttmann, T. Buchner, S. Werner, G. Laube, A. Hornemann, B. Tarek, G. Schneider and J. Kneipp, Nanoscale, 2013, 5, 9193-9198.

17. D. A. H. Hanaor, M. Michelazzi, C. Leonelli and C. C. Sorrell, Journal of the European Ceramic Society, 2012, 32, 235-244.

18. D. Aherne, D. E. Charles, M. E. Brennan-Fournet, J. M. Kelly and Y. K. Gun'ko, Langmuir, 2009, 25, 1016510173.

19. B. Pelaz, V. Grazu, A. Ibarra, C. Magen, P. del Pino and J. M. de la Fuente, Langmuir, 2012, 28, 8965-8970.

20. E. N. Esenturk and A. R. HightWalker, J. Raman Spectrosc. , 2009, 40, 86-91.

21. C. G. Khoury and T. Vo-Dinh, The journal of physical chemistryC, Nanomaterials and interfaces 2008, 112, 18849-18859.

22. S. M. Ali, F. Bonnier, A. Tfayli, H. Lambkin, K. Flynn, V. McDonagh, C. Healy, T. Clive Lee, F. M. Lyng and H. J. Byrne, J Biomed Opt, 2013, 18, 061202.

23. J. Vura-Weis, M. D. Newton, M. R. Wasielewski and J. E. Subotnik, J. Phys. Chem. C 2010, 114, 20449-20460.

24. A. Kudelski, Chemical Physics Letters 2005, 414, 271275.

25. R. Narayanan, R. J. Lipert and M. D. Porter, Anal. Chem., , 2008, 80 2265-2271.

26. Q. Yu, P. Guan, D. Qin, G. Golden and P. M. Wallace, Nano Lett, 2008, 8, 1923-1928.

27. Félidj N, Aubard J, Lévi G, Krenn JR, Salerno M, Schider G, Lamprecht B, Leitner A and A. FR, Phys Rev B 2002, 65, 075419.

$28 . \quad$ J. E. Millstone, S. J. Hurst, G. S. Metraux, J. I. Cutler and C. A. Mirkin, Small, 2009, 5, 646-664.

29. R. Jin, Y. Cao, C. A. Mirkin, K. L. Kelly, G. C. Schatz and J. G. Zheng, Science, 2001, 294, 1901-1903.

30. G. Plascencia-Villa, D. Bahena, A. R. Rodriguez, A. Ponce and M. Jose-Yacaman, Metallomics, 2013, 5, 242-250.
31. X. N. He, Y. Gao, M. Mahjouri-Samani, P. N. Black, J. Allen, M. Mitchell, W. Xiong, Y. S. Zhou, L. Jiang and Y. F. Lu, Nanotechnology 2012, 23, 205702

32. D. Zhang, S. M. Ansar, K. Vangala and D. Jiang, Journal of Raman Spectroscopy 41 :952-957., 2010, 41, 952-957.

33. J. S. Gebauer, M. Malissek, S. Simon, S. K. Knauer, M. Maskos, R. H. Stauber, W. Peukert and L. Treuel, Langmuir, 2012, 28, 9673-9679.

34. A. Bonifacio, S. Dalla Marta, R. Spizzo, S. Cervo, A. Steffan, A. Colombatti and V. Sergo, Anal Bioanal Chem, 2014, 406, 2355-2365.

35. G. Braun, S. Lee, T. Laurence, N. Fera, L. Fabris, G. C. Bazan, M. Moskovits and N. O. Reich, J Phys Chem C, 2009, 113, 13622-13629.

36. K. L. Wustholz, A. I. Henry, J. M. McMahon, R. G. Freeman, N. Valley, M. E. Piotti, M. J. Natan, G. C. Schatz and R. P. Van Duyne, J Am Chem Soc, 2010, 132, 10903-10910.

37. T. A. Laurence, G. B. Braun, N. O. Reich and M. Moskovits, Nano Lett., 2012, 12, 2912-2917.

38. L. J. Sherry, R. Jin, C. A. Mirkin, G. C. Schatz and R. P. Van Duyne, Nano Lett, 2006, 6, 2060-2065.

39. P. Mulvaney, Langmuir, , 1996, 12 788-800.
40. S. L. Kleinman, R. R. Frontiera, A. I. Henry, J. A. Dieringer and R. P. Van Duyne, Phys Chem Chem Phys 2013, 15, 21-36.

41. J. H. Seo, H. S. Park, Y. Yoo, T. Y. Seong, J. Li, J. P. Ahn, B. Kim and I. S. Choi, Nano Lett, 2013, 13, 5112-5116.

P. S. Kumar, I. Pastoriza-Santos, B. Rodriguez-Gonzalez, F. J. G. de Abajo and L. M. Liz-Marzan, Nanotechnology 2008, 19, 015606.

43. J. Rodríguez-Fernández, I. Pastoriza-Santon, J. PérezJuste, F. J. G. d. Abajo and a. L. M. Liz-Marzán, J. Phys. Chem. C, 2007, 111, 13361-13366.

44. A. Wei, B. Kim, B. Sadtler and S. L. Tripp, ChemPhysChem, 2001, 2, 743-745.

45. J. Gersten and A. Nitzan, J Chem Phys 1980, 73, 30233037.

46. X. Zhang, C. R. Yonzon, M. A. Young, D. A. Stuart and R. P. Van Duyne, IEE Proc., Nanobiotechnol. , 2005, 152, 195.

47. J. Hu, Z. Wang and J. Li, Sensors (Basel), 2007, 7, 3299 3311.

48. C. E. Talley, J. B. Jackson, C. Oubre, N. K. Grady, C. W. Hollars, S. M. Lane, T. Huser, P. Nordlander and N. J. Halas, Nano Lett, 2005, 5, 1569-1574. 